\title{
Supplementary Information: Molecular mechanisms of activation in CDK2
}

\author{
Neva Besker, ${ }^{1}$ Andrea Amadei, ${ }^{1}$ and Marco D'Abramo*2 \\ ${ }^{1}$ Dipartimento di Scienze e Tecnologie Chimiche, Universita di Roma Tor Vergata, \\ Via della Ricerca Scientifica, 1, 00185, Rome, Italy \\ ${ }^{2}$ CINECA Roma, Via dei Tizii 6, 00185, Rome, Italy*
}

(Dated: July 23, 2013)

*mdabramo.res@gmail.com 
TABLE I: RMSD values

\begin{tabular}{l|c|c|c} 
Simulation & N. eigenvectors & RMSD (nm) & reference structure \\
\hline EDS & all & 0.05 & target \\
EDS & 50 & 0.17 & target \\
EDS & 20 & 0.25 & target \\
EDS & 10 & 0.31 & target \\
EDS & 10 & 0.31 & target \\
MD free (closed) & n.a. & 0.24 & starting \\
MD free (open) & n.a. & 0.22 & starting
\end{tabular}

EDS means Essential Dynamics Sampling simulations. The statistical error on the RMSD values is estimated to be less than $0.02 \mathrm{~nm}$.

TABLE II: Secondary Structures

\begin{tabular}{l|c|c} 
Simulation & N. of structured residues (max-min) & std. dev. \\
\hline EDS opening (final structures) & $160-177$ & 6.5 \\
EDS closure (final structures) & $165-180$ & 6.4 \\
MD free (closed) & $165-180$ & 5.1 \\
MD free (open) & $170-180$ & 4.6
\end{tabular}

Number of structured residues considering the last 10 ps of the EDS simulations and the corresponding target and starting structures used in the EDS simulations.

\section{SUPPLEMENTARY INFORMATION}




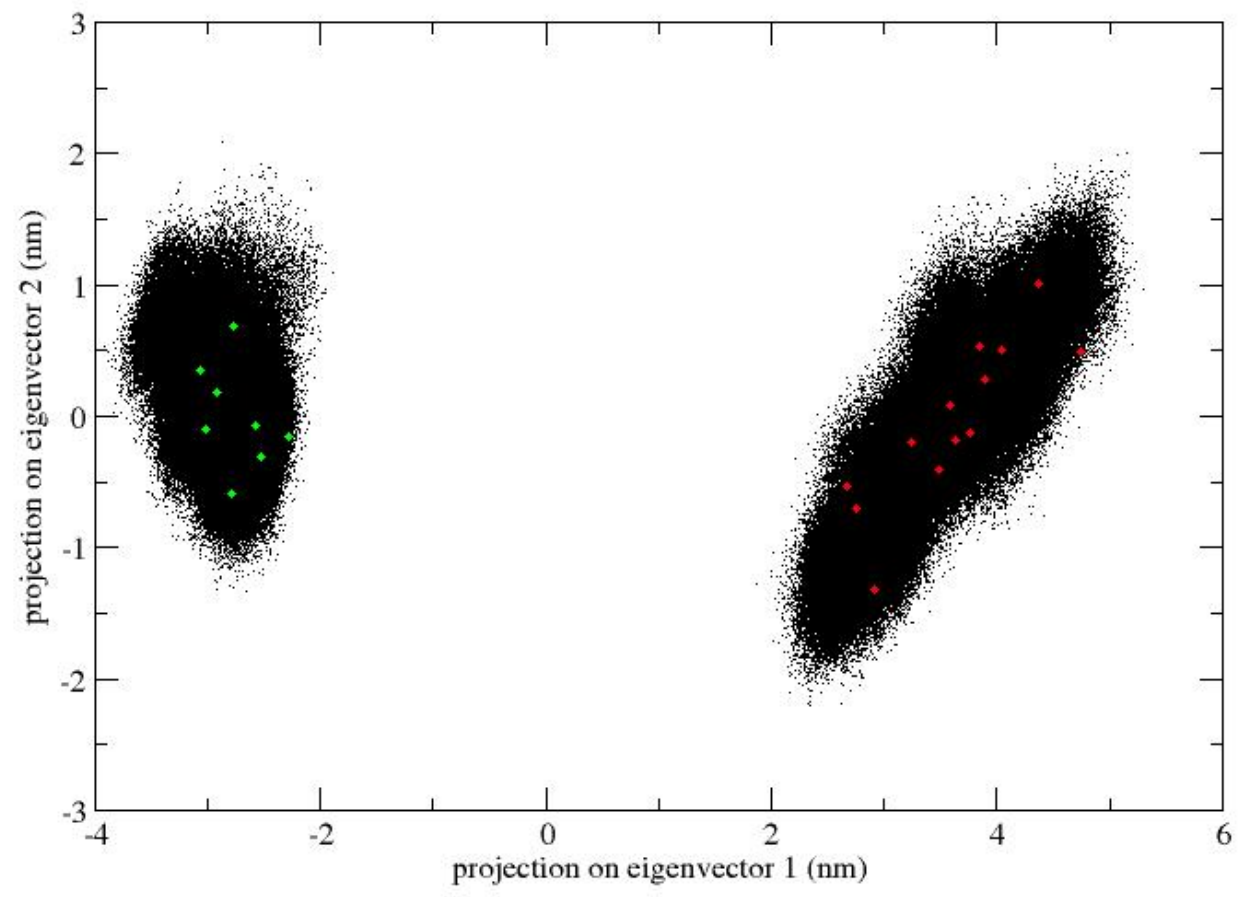

Supplementary Figure 1: Projection of the free molecular dynamics trajectories of the CDK2 open (on the right) and closed (on the left) forms. The projections of the selected initial structures for the EDS techniques are represented by green (red) marks for the closed (open) conformational state. 

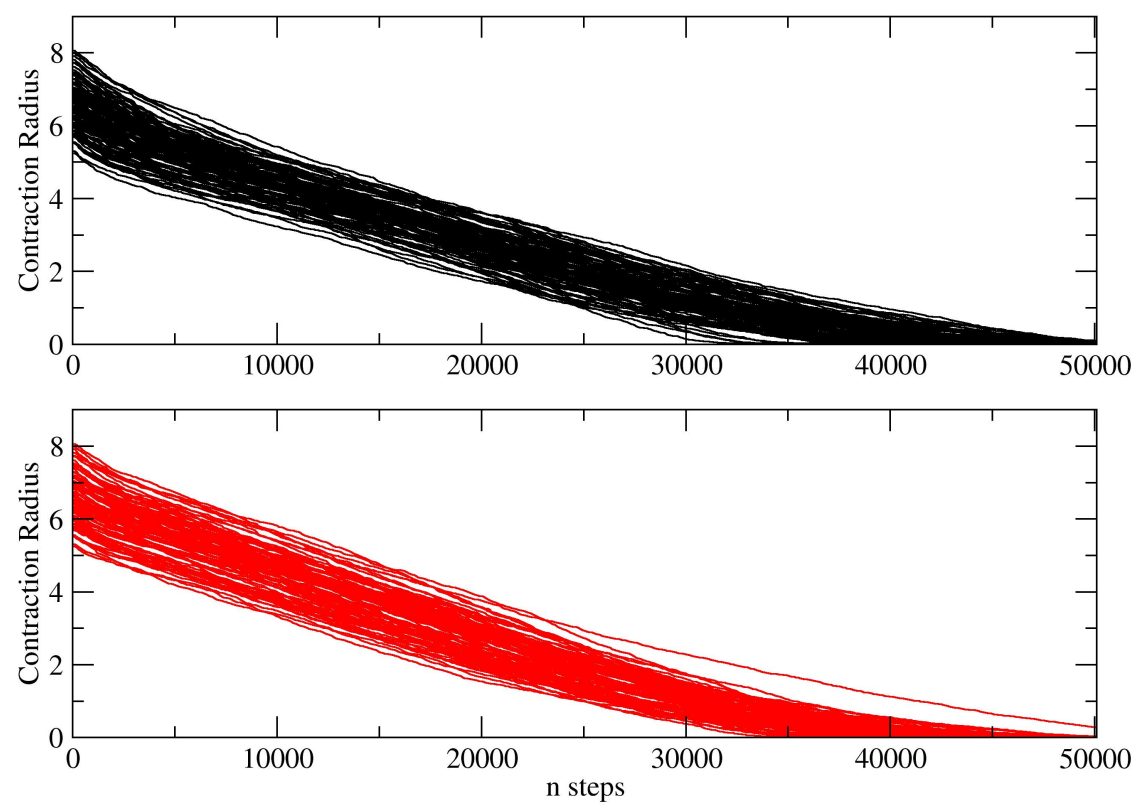

Supplementary Figure 2: Contraction radius versus the number of EDS step for the closure (top panel) and for the opening process (bottom panel). 

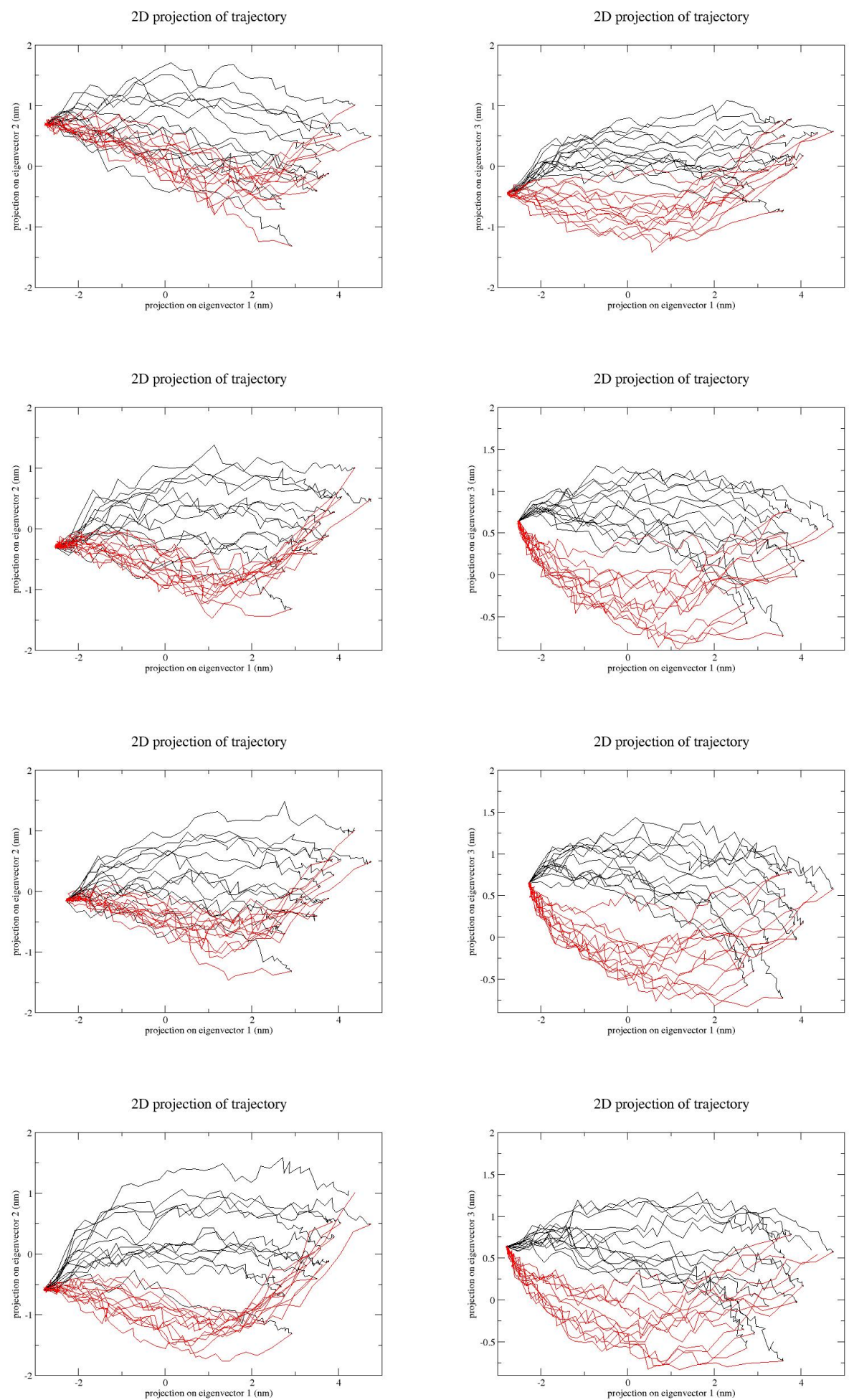

Supplementary Figure 3: Projections of the EDS trajectories (red lines: open-to-closed; black lines: closed-to-open) on the essential subspaces (left column: eigenvector 1 vs 2; right column: eigenvector 1 vs 3 ). 

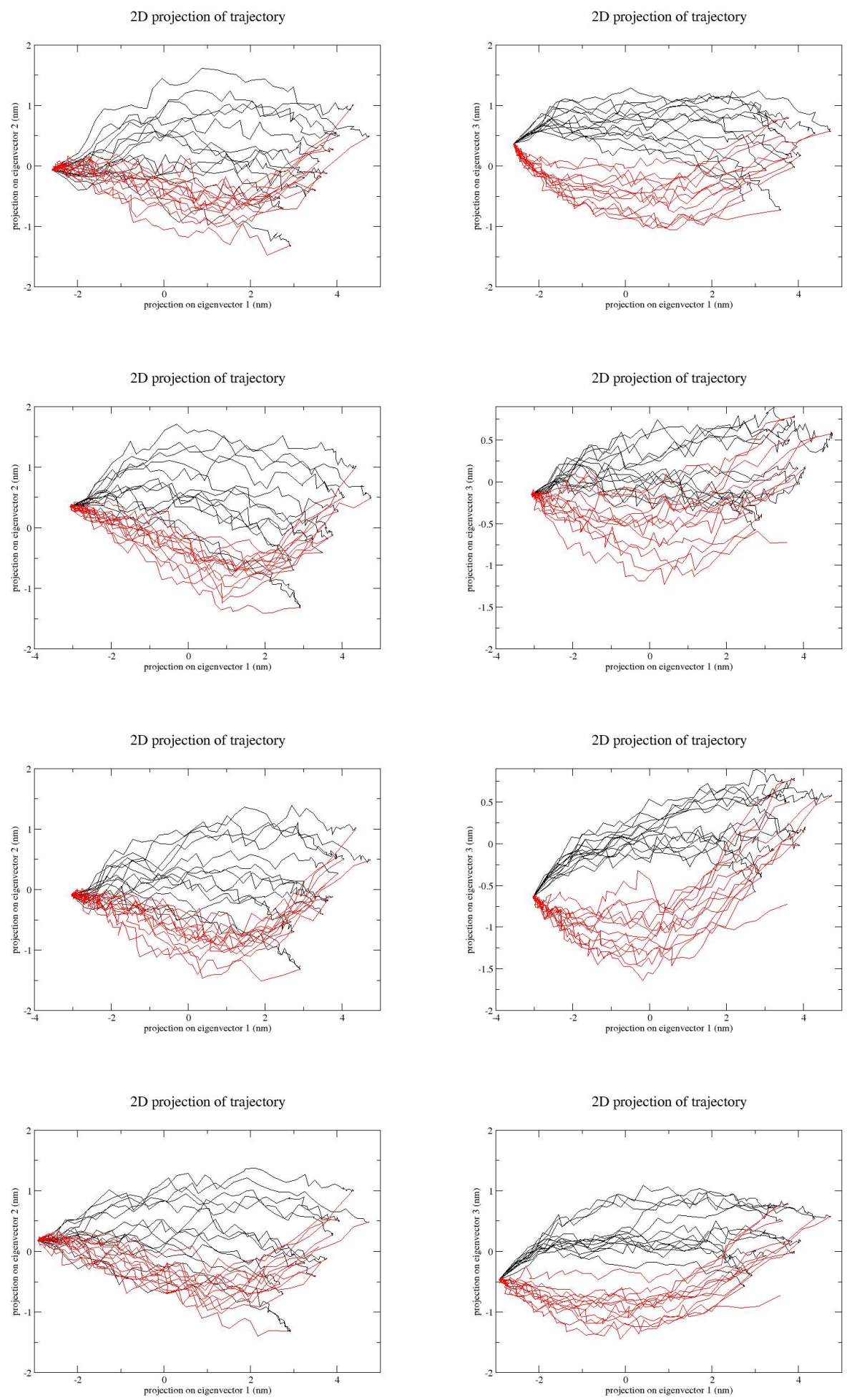

Supplementary Figure 4: Projections of the EDS trajectories (red lines: open-to-closed; black lines: closed-to-open) on the essential subspaces (left column: eigenvector 1 vs 2; right column: eigenvector 1 vs 3 ). 

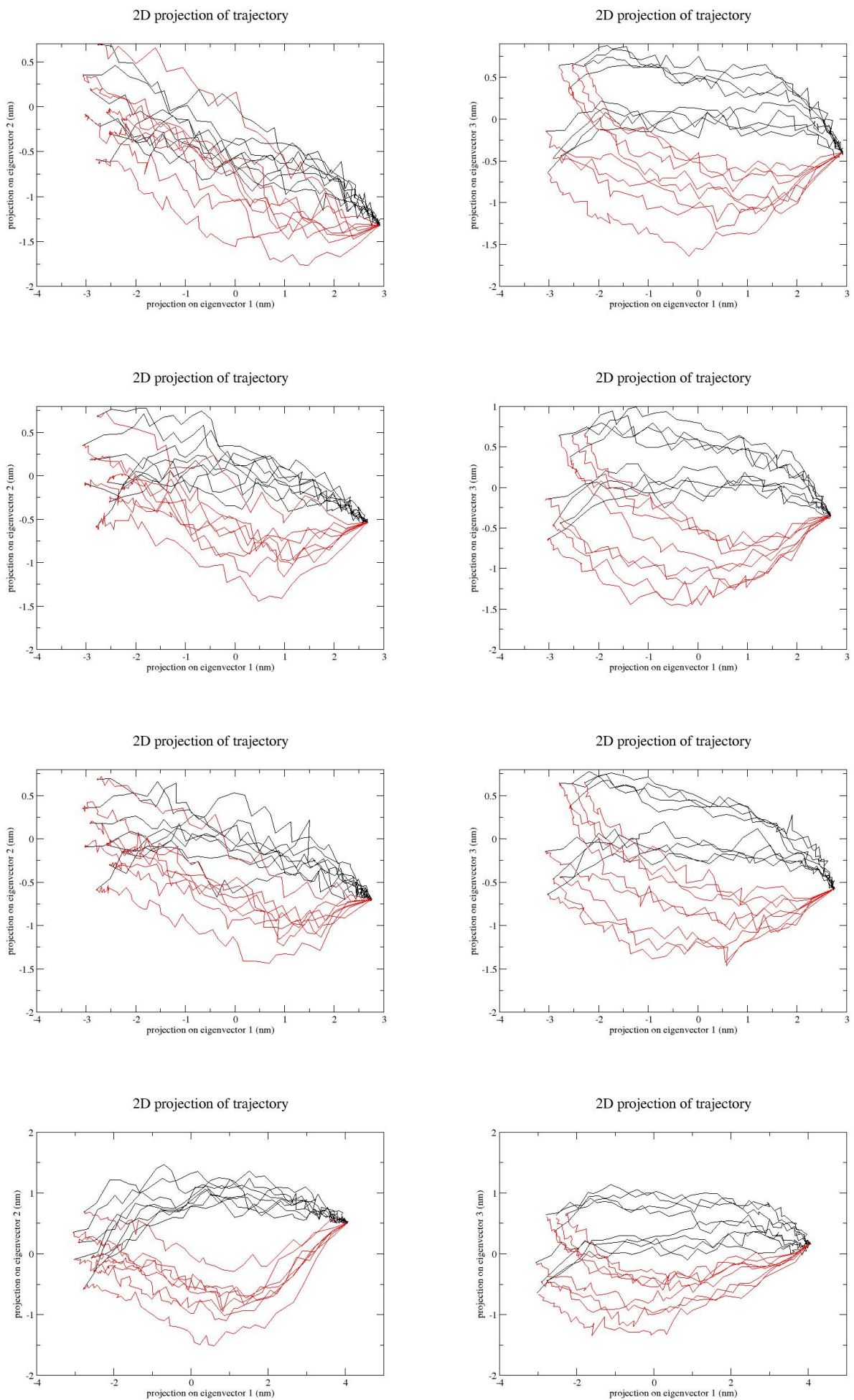

Supplementary Figure 5: Projections of the EDS trajectories (red lines: open-to-closed; black lines: closed-to-open) on the essential subspaces (left column: eigenvector 1 vs 2; right column: eigenvector 1 vs 3). 

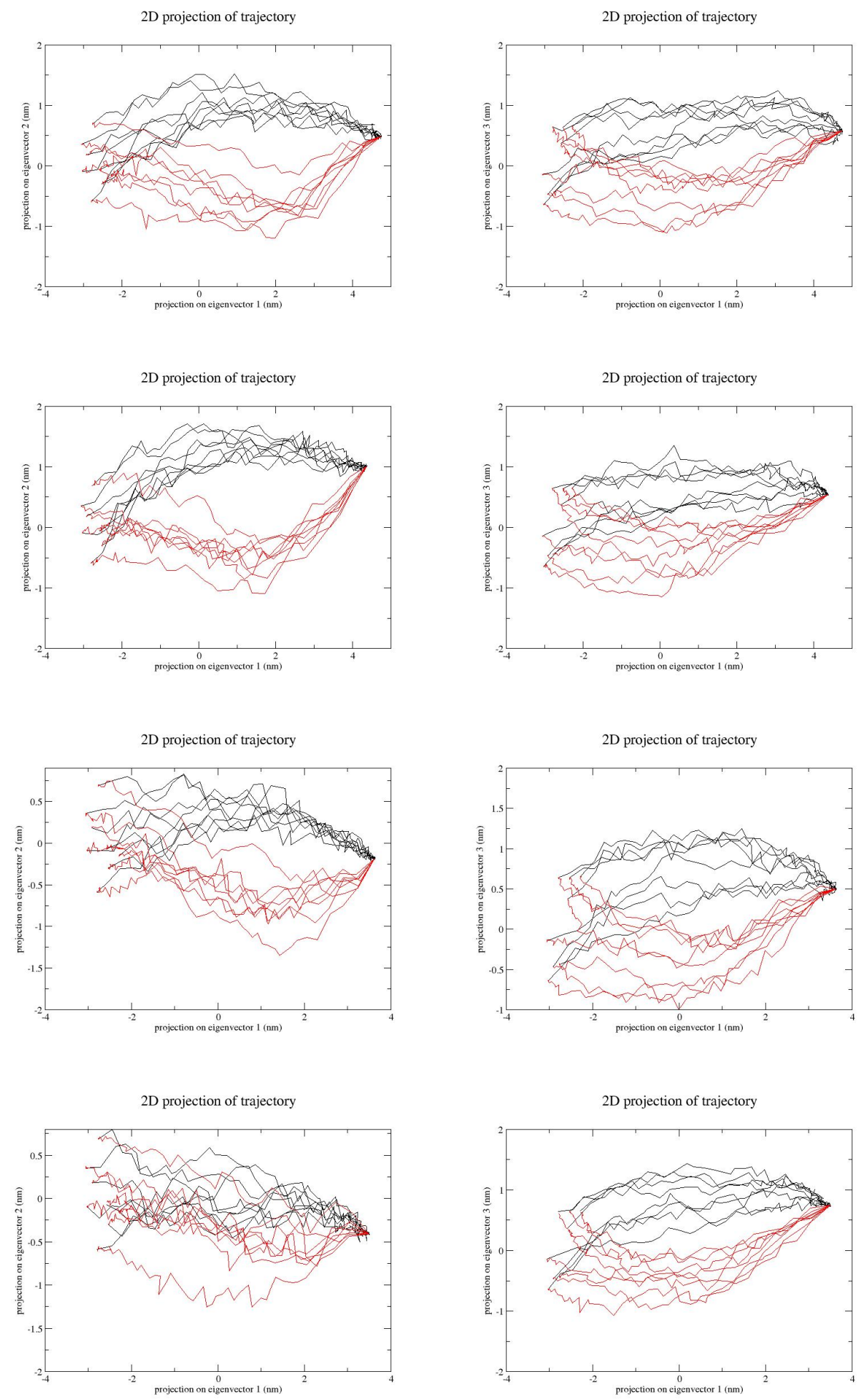

Supplementary Figure 6: Projections of the EDS trajectories (red lines: open-to-closed; black lines: closed-to-open) on the essential subspaces (left column: eigenvector 1 vs 2 ; right column: eigenvector 1 vs 3 ). 